\title{
TEM Study of Aluminum Oxynitride Films Prepared by Reactive R.F.-Sputtering
}

\author{
Li-Chung Lai", Masateru Nose ${ }^{* *}$, Wen-An Chiou ${ }^{*}$ and Atsushi Saiki*** \\ * NISP Lab, NanoCenter, University of Maryland, College Park, MD 20742-2831 \\ **Faculty of Art and Design, Univ. of Toyama, 180 Futagami-machi, Takaoka 933-8588, Japan \\ *** School of Science and Engineering, Univ. of Toyama, 3190 Gofuku, Toyama 930-8555, Japan
}

The versatile of physical properties of Aluminum Oxynitride $\left(\mathrm{AlO}_{\mathrm{X}} \mathrm{N}_{\mathrm{Y}}\right)$ offers many applications in various technologies, e. g., microelectronics, optics and protective coatings [1-3]. $\mathrm{AlO}_{\mathrm{X}} \mathrm{N}_{\mathrm{Y}}$ films are generally prepared by reactive sputtering in a mixture of nitrogen and oxygen $\left(\mathrm{N}_{2}+\mathrm{O}_{2}\right)$ gases [4] with aluminum target. However, control of the oxygen concentration in the reactive sputtering system is difficult due to the high reactivity of oxygen to aluminum. To improve the reactive sputtering system, a modified reactive rf-sputtering using $\mathrm{N}_{2}+$ Ar gas mixture with $\mathrm{Al}_{2} \mathrm{O}_{3}$ target was initiated. This study reports the microstructure of $\mathrm{AlO}_{\mathrm{X}} \mathrm{N}_{\mathrm{Y}}$ films obtained by this new method.

$\mathrm{Al}_{2} \mathrm{O}_{3}$ (99.6\%) target (Nikkato Co., Japan) was sputtered using a facing target-type sputtering system (FTS-2R Model, Osaka Vacuum Co.) with $980 \mathrm{~W}$ power in a mixture of highly purified Ar and $\mathrm{N}$ gases (99.9999\%) with the flow rate of 10 and $30 \mathrm{sccm}$ (standard cubic centimeter per minute), respectively. Si substrates were preheated to $350{ }^{\circ} \mathrm{C}$ with a bias of $-100 \mathrm{~V}$ during the deposition. Total thickness of the film was approximately $2 \mu \mathrm{m}$. Cross-sectional TEM specimens were prepared using the FIB (Focus Ion Beam) technique. Plane-view TEM specimens were prepared by mechanical griding/poilishing followed by ion milling at $-60^{\circ} \mathrm{C}$ with a final accelerating voltage at $0.5 \mathrm{kV}$. Film micro-structure and -chemistry were examined by JEM 2100 FEGTEM. Detail chemical composition was analyzed by JEOL EPMA.

Figure 1 shows the change in indentation hardness $\left(H_{p l}\right)$ of the films produced at different biases and at different substrate temperatures. The indentation hardness increases with increasing bias as well as with substrate temperature. The softest film deposited at room temperature without substrate bias showed an amorphous-like and/or poly-nanocrystalline XRD pattern (Fig. 2a). However, the hardest film deposited at $350{ }^{\circ} \mathrm{C}$ with a substrate bias of $-100 \mathrm{~V}$ revealed a crystalline structure (Fig. $2 \mathrm{~b}$ ); this is also evidenced by the HRTEM and diffraction contrast images (Figs. 3 and 4). The cross-sectional TEM dark-field image also depicts that the elongated columnar crystal strucuture of film is parallel to the direction of film growth (Fig. 3). An armorphous interlayer was found at the interface between the film and Si substrate (Fig. 5). The electron diffraction patterns obtained from the film (Fig. 4) and the interface region (Fig. 6) suggest that the film consists mainly of hexagonal AlN (JCPDS 25-1133) poly-nanocrystallites with some degrees of preferred crystal growth orientation. In addition to the presence of $\mathrm{Al}$ and $\mathrm{N}$ in the film, a high concentration of oxygen $(\sim 10 \%$ or more) was found in the EPMA and TEM EDS results. Of particular interest, AlN was the only oxygenated material that matched well with the diffraction pattern, yet oxygen was found evenly distributed throughout the film. This finding is difficult to interpret, but one possible explanation is that the film was composed mainly of nanocrystalline AlN with small amounts of $\mathrm{AlOx}$ or $\mathrm{AlO}_{\mathrm{X}} \mathrm{N}_{\mathrm{Y}}$. Further investigation is in progress to confirm the speculation and clarify the behavior of oxygen during the process of film deposition.

[1] S. Dreer, R. Krismer, P.Wilhartitz, G. Friedbacher, Thin Solid Films 354(1999) 43.

[2] C. K. Hwangbo, L. J. Lingg, J. P. Lehan, H. A. Macleod, F. Suits, Appl. Opt. 28(1989) 2779.

[3] H. Birey, S. J. Pak, J. R. Sites, J. F.Wager, J. Vac. Sci. Technol. 16(1979) 2086.

[4] S. Dreer, Fresenius' Journal of Analytical Chemistry, 365 (1999) 85. 
[5] TEM work at NISP Lab was partially supported by NSF-MRSEC (DMR 05-20471) and UMD.
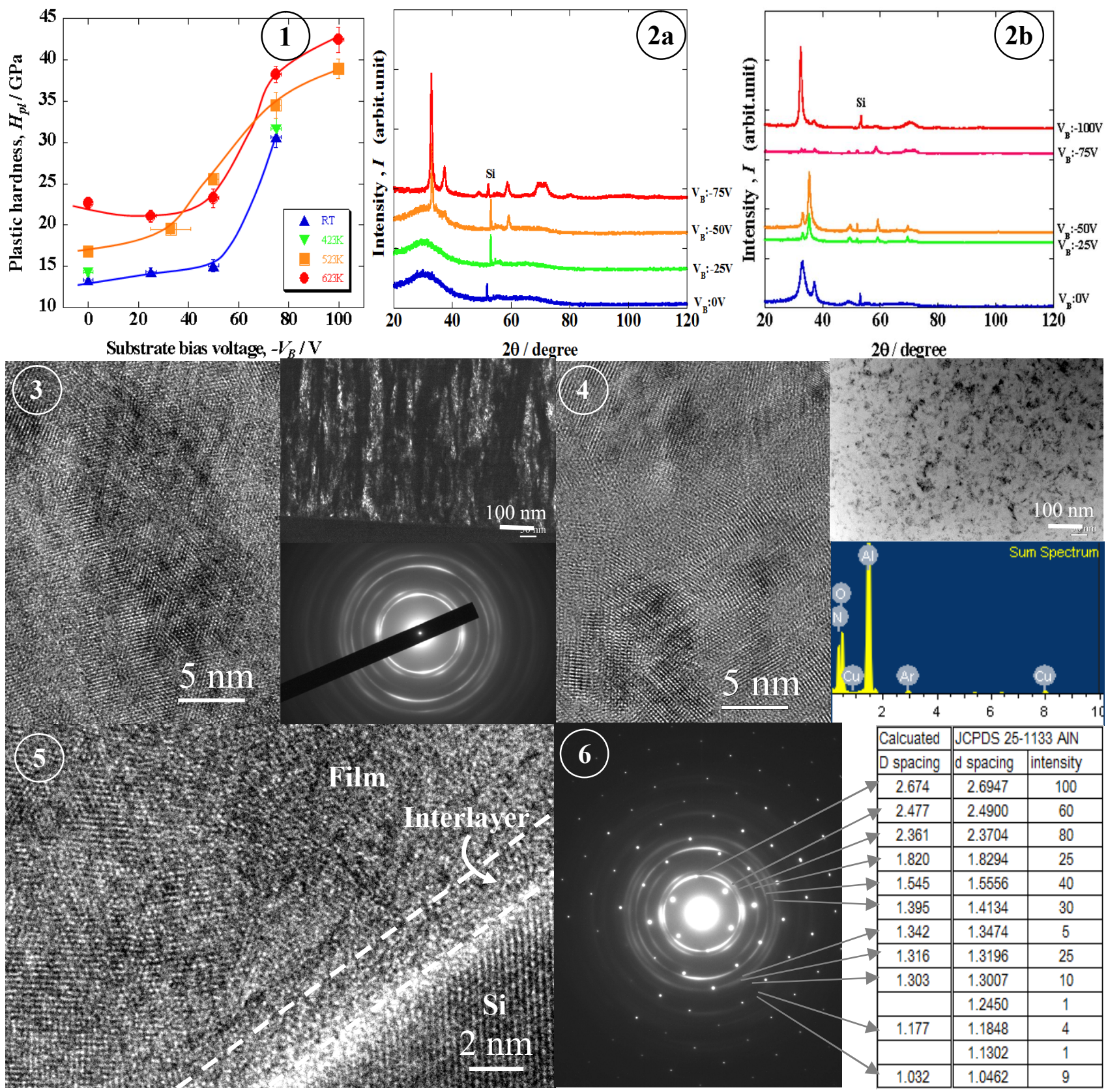

Fig. 1. Diagram shows plastic hardness $\left(\mathrm{H}_{\mathrm{pl}}\right)$ of film with different bias voltage and substrate temperature.

Fig. 2. XRD patterns of films deposited on different substrate biases: at $25^{\circ} \mathrm{C}(2 \mathrm{a})$ and $350{ }^{\circ} \mathrm{C}(2 \mathrm{~b})$.

Fig. 3. Cross-sectional DF and HRTEM images of the film show poly-nanocrystalline columnar structure along the film growth direction. SAD pattern matches with typical AIN (Fig. 6).

Fig. 4. Plane-view BF and HRTEM images of the film show a typical poly-crystalline structure. EDS spectrum showing $\mathrm{Al}, \mathrm{N}$ and $\mathrm{O}$ throughout the film.

Fig. 5. Cross-sectional HRTEM image shows an amorphous interlayer at film $/ \mathrm{Si}$ interface.

Fig. 6. Analysis of SAD pattern obtained from film/Si interface area (Fig. 5) reveals the film is composed mainly of nanocrystalline AIN. Diffraction spots are contributed from Si substrate. 